\title{
Fully covered self-expanding metal stents for refractory pancreatic duct strictures in symptomatic chronic pancreatitis, US experience
}

다(1)이우

\author{
Authors \\ Institutions \\ 1 Weill Cornell Medicine, Division of Gastroenterology and \\ Hepatology, Department of Medicine, New York, New \\ York, United States \\ 2 Thomas Jefferson University Hospital, Division of \\ Gastroenterology and Hepatology Philadelphia, \\ Pennsylvania, United States \\ 3 Stony Brook University Medicine, Division of \\ Gastroenterology and Hepatology, Stony Brook, New \\ York, United States \\ 4 Icahn School of Medicine at Mount Sinai, Division of \\ Gastroenterology, New York, New York, United States \\ 5 Geisinger Health System, Danville Pennsylvania, United \\ States
}

Reem Z. Sharaiha ${ }^{*}, 1$, Aleksey Novikov ${ }^{*},{ }^{1,2}$, Kristen Weaver ${ }^{1}$, Pawan Marfatia', Jonathan M. Buscaglia ${ }^{3}$, Christopher J. DiMaio $^{4}$, David Diehl ${ }^{5}$, Moamen M. Gabr ${ }^{1}$, Monica Gaidhane ${ }^{1}$, Ali Siddiqui ${ }^{2}$, Michel Kahaleh ${ }^{1}$

submitted 29.5.2018

accepted after submission 25.1.2019

Bibliography

DOI https://doi.org/10.1055/a-0858-2169 |

Endoscopy International Open 2019; 07: E1419-E1423

(c) Georg Thieme Verlag KG Stuttgart · New York

eISSN 2196-9736

Corresponding author

Reem Sharaiha, MD, MSc, Associate Professor of Medicine, Director of Bariatric \& Metabolic Endoscopy, Associate Director of the Pancreas Program, Weill Cornell Medicine, Division of Gastroenterology and Hepatology, Department of Medicine, 1305 York Avenue, 4th Floor, New York, New York 10021

Fax: +1-646-962-0110

rzs9001@med.cornell.edu

\section{ABSTRACT}

Background and study aims Fully covered self-expanding metal stents (FCSEMS) have been used to treat refractory pancreatic duct strictures. We aimed to evaluate the feasibility, safety, and efficacy of FCSEMS in chronic pancreatitis with refractory pancreatic duct strictures.

Patients and methods This was a retrospective multicenter cases series of patients who underwent endoscopic retrograde cholangiopancreatography (ERCP) with FCSEMS placement in the main pancreatic duct (MPD) at five tertiary care centers between February 2010 and June 2016. Primary endpoints were technical success, clinical success, and procedure-related morbidity. Secondary endpoints were pain relief at the end of follow-up and resolution of the pancreatic stricture on ERCP.

Results Thirty-three patients with previously drained stents, $76 \%$ of whom were male, underwent ERCP with FCSEMS placement. Mean duration of follow-up was 14 months. All of the patients had prior therapy. The technical success rate for FCSEMS placement was $100 \%(n=33)$ and the clinical success rate was $93 \%$ (was $n=31$ ). Stents were removed after a median duration of 14.4 weeks. After stent removal, the diameter of the narrowest MPD stricture had increased significantly from $1 \mathrm{~mm}$ to $4.5 \mathrm{~mm}(P<0.001)$. There was a statistically significant improvement on the Visual Analogue Scale (VAS) from a median of 8.5 to 2.5. At the end of the study, $(n=27) 87.1 \%$ of patients reported significant pain reduction with reduced narcotic use.

Conclusion FCSEMS appeared to be a feasible, safe, and potentially effective Intervention in patients who had not responded to endoscopic therapy with plastic stents.

\section{Introduction}

Chronic pancreatitis (CP) is a fibro-inflammatory disease of the pancreas that often is a result of heavy and prolonged alcohol use. It can also occur as a final consequence of pancreaticobili- ary obstruction, cystic fibrosis, autoimmune pancreatitis, or other rheumatologic diseases [1]. In a single-center study, CP had a sex- and age-adjusted prevalence of 42 cases per 100,000 person years [2]. It results in more than 122,000 outpatient visits and in more than 56,000 hospitalizations yearly in the United States alone [2]. CP manifests as both exocrine and

* These authors contributed equally. 

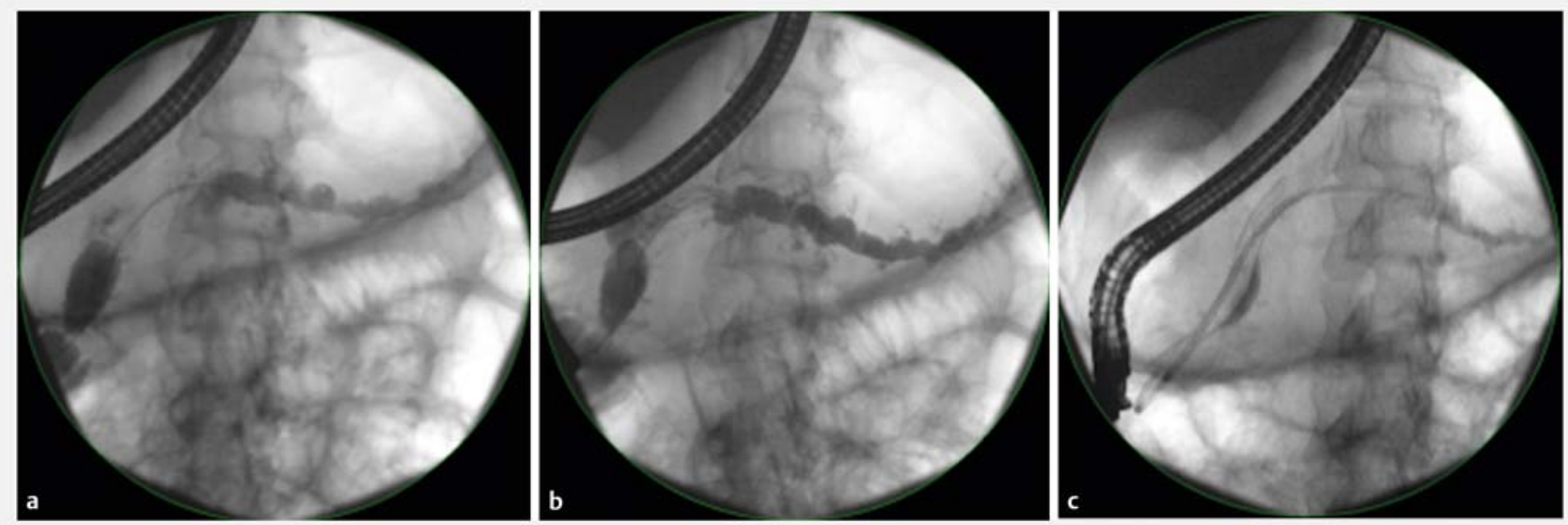

Fig. 1 a PD cannulation with a guidewire. b Balloon dilation of the pancreatic duct. c Placement of the FCSEMS into the PD with an anchoring double pigtail stent

endocrine insufficiency. It is characterized by strictures and progressive fibrosis with destruction of the islet and acinar cells [3]. Severe episodes of pain are the presenting complaint in $30 \%$ to $85 \%$ of patients [4], causing disability, early retirement, opiate addiction, and recurrent hospitalizations [5], Symptomatic pancreatic duct strictures are commonly managed endoscopically with plastic stents. However, up to $68 \%$ of treated patients still experience pain 5 years post-intervention. The Dutch multicenter trial showed that conventional endoscopic management with plastic stents and balloon dilation had a lower rate of success than longitudinal pancreaticojejunostomy [6]. Fully covered self-expanding metal stents (FCSEMS) have been used successfully to treat benign biliary strictures, and have also been reported to be useful in management of pancreatic duct strictures [3]. The aim of this study was to investigate the feasibility, safety, and efficacy of FCSEMS in refractory pancreatic duct strictures.

\section{Patients and methods}

A retrospective review of patients with moderate to severe chronic pancreatitis who had undergone endoscopic retrograde cholangiopancreatography (ERCP) with FCSEMS placement between February 2010 and June 2016 at five tertiary care centers was performed. Patients were included if they had refractory pain despite prior treatment with a conventional therapy that included at least three balloon dilations, repeat plastic stent placements with upsizing of the stents, or stone lithotripsy. Patients with malignant strictures were excluded.

Technical success was defined as successful placement of the FCSEMS during the ERCP. Clinical success was defined as improvement in stricture diameter as well as symptomatic pain relief seen by improvement in pain scores on the visual analogue scale (VAS). Adverse events (AEs), including infection, stent occlusion, stent migration, and bleeding were noted. AEs were graded according to the American Society for Gastrointestinal Endoscopy lexicon severity grading system [5,7]. As this was a retrospective study, clinical success and AEs were documented by each institution in various forms by either phone or office visit. All patients gave informed consented for the procedure and all centers received approval by their respective institutional review board.

\section{Endoscopic technique}

Endoscopists who performed the endoscopic procedures all had extensive experience in endoscopic management of chronic pancreatitis, each having done more than 1000 ERCPs. Sideviewing endoscopes (TJF-180, TJF-160, Olympus America, Center Valley, Pennsylvania, United States) were used for all procedures. Pancreatic sphincterotomy was performed on all patients before FCSEMS placement. The pancreatic stricture was dilated with either a 4-mm or 6-mm balloon dilator (Hurricane, Boston Scientific, Natick, Massachusetts, United Sates), then, over a 0.035-inch guide- wire, an 8-mm-diameter or 10-mm-diameter FCSEMS (Wallflex, Boston Scientific Corp, Natick, Massachusetts, United States) was placed in the main PD across the stricture ( $\triangleright$ Fig. 1 ); the length of the stent was determined by the length of the stricture. Biliary sphincterotomy was performed at the same time if no prior one was performed. The FCSEMSs were removed after a period of at least 3 weeks with snare or rat-tooth forceps and pressure injection was performed to confirm pancreatic drainage after stent removal.

\section{Outcome measures}

Primary endpoints were technical success, clinical success, and procedure-related morbidity. Secondary endpoints were pain relief at the end of follow-up, resolution of the pancreatic stricture on fluoroscopy during ERCP and AEs. Pain scores were collected prior to the procedure and in follow-up on most patients, as well as doses of analgesic medications at each office visit or over phone. Pain scores were recorded as VAS if available at each office visit. 


\begin{tabular}{|c|c|c|}
\hline \multicolumn{3}{|l|}{ Demographics $n=33$ (\%) } \\
\hline $\begin{array}{l}\text { Age, years median } \\
\text { (range) }\end{array}$ & & $\begin{array}{l}54 \text { years } \\
(18-87)\end{array}$ \\
\hline Male sex & & $25(75.8)$ \\
\hline \multirow{3}{*}{$\begin{array}{l}\text { Prior endoscopic treat- } \\
\text { ment }\end{array}$} & Balloon dilation & $20(60.6)$ \\
\hline & Plastic stent placement & $33(100)$ \\
\hline & $\begin{array}{l}\text { ESWL } \\
\text { Lithotripsy }\end{array}$ & $\begin{array}{l}8(24.2) \\
17(51.5)\end{array}$ \\
\hline \multirow[t]{4}{*}{ Etiology } & Alcohol & $19(57.6)$ \\
\hline & Idiopathic & $8(24.2)$ \\
\hline & Divisum & $2(6.06)$ \\
\hline & Unknown & $4(12.1)$ \\
\hline Diabetes & & $5(15.1)$ \\
\hline Exocrine Insufficiency & & $29(87.8)$ \\
\hline \multicolumn{3}{|l|}{ Outcomes } \\
\hline Technical success rate & & $33(100)$ \\
\hline \multicolumn{3}{|l|}{ Adverse events } \\
\hline & Overall & $11(33$ \\
\hline & $\begin{array}{l}\text { Immediate Stent } \\
\text { Removal }\end{array}$ & $2(6.06)$ \\
\hline & $\begin{array}{l}\text { Cholestasis after stent } \\
\text { placement }\end{array}$ & $2(6.06)$ \\
\hline & Stent impaction & $1(3.03)$ \\
\hline & $\begin{array}{l}\text { Worsening abdominal } \\
\text { pain }\end{array}$ & $6(18.2)$ \\
\hline & Migration & $0(0.00)$ \\
\hline \multicolumn{2}{|c|}{ Stent duration, weeks median (range) } & $15(12-22)$ \\
\hline Reduction in narcotic use & & $27(87.1)$ \\
\hline Need for further therapy & & $12(36.4)$ \\
\hline
\end{tabular}

\section{Statistical analysis}

Descriptive statistics were calculated for all demographic and clinical variables and reported as mean \pm standard deviation, median (interquartile range), or as a proportion where appropriate. Univariate analysis was performed using $x^{2}$ test and Fisher exact test for categorical variables and Student's $t$ test, Wilcoxon or Mann-Whitney $U$ test as required for continuous variables. All variables were tested for normality using the Shapiro-Wilk test. All statistical analysis was conducted using STATA 13.0 (StataCorp LP, College Station, Texas, United States). $P<0.05$ was considered significant.



- Fig. 2 Tukey plot showing decrease in VAS after FCSEMS intervention. Whiskers extend to $1.5^{*}$ interquartile range

\section{Results}

A total of 33 patients ( $75 \%$ male, mean age $53.9 \pm 13.1$ years) with chronic symptomatic pancreatitis underwent ERCP with a FCSEMS (Wallflex, Boston Scientific, Natick, Massachusetts, United States) placement with a mean follow-up time of 14 months (range: 6-24). Strictures were located in the head, genu or proximal body in all patients. Pancreatic stones were present in 17(51\%) patients. Prior therapy included balloon dilation and plastic stent placement in all 33 patients (100\%), mechanical and laser lithotripsy in 17 patients (51\%) as well as extracorporeal shockwave lithotripsy (ESWL) therapy in eight patients $(24 \%)$. ( Table 1$)$. The underlying etiology for pancreatitis included alcohol $(n=19,58 \%)$, idiopathic $(n=8,24 \%)$, divisum $(n=2,8 \%)$, and other $(n=4,12 \%)$. Four patients also had a stent in the common bile duct (CBD): FCSEMSs in two patients and plastic stents in the other two patients. One patient had a FCSEMS placed through his minor papilla while the remainder was placed through the major papilla. Stents were removed after median of 14.4 weeks (range 12 - 22).

\section{Technical and clinical success rates}

Technical success was achieved in all 33 patients (100\%). Clinical success with successful subjective pain relief was achieved in 31 patients (93\%). Objective long-term pain relief with reduction in pain medication was achieved in 27 patients (87\%) ( Table 1). Median patient pain score prior to the procedure on the VAS was 8.5 (range $7.25-9.75$ ) and post procedure was 2.5 (range $1-3$ ) with a statistically significant improvement $(P$ $<0.001$ ) ( $>$ Fig. 2). After stent removal, the diameter of the narrowest MPD stricture had increased significantly from $1 \mathrm{~mm}$ to $4.5 \mathrm{~mm}(P<0.001)$.

No new strictures developed in anyone. Twelve patients (36\%) required further therapy, while the remainder became stent free. Four patients were stent dependent and two of them required eventual surgery. 
- Table 2 Publications with metal stent placement for chronic pancreatitis.

\begin{tabular}{|c|c|c|c|c|}
\hline Author & Patients & Success & Complications & Outcomes \\
\hline Sauer et al. [12] & 6 & $50 \%$ & None & $\begin{array}{l}\text { Temporary placement of FSEMSs with fins in patients with } \\
\text { symptomatic refractory PD stricture offered transient relief } \\
\text { of pain }\end{array}$ \\
\hline Park et al. [13] & 13 & $69 \%$ & $\begin{array}{l}2 \text { cases of acute pancreatitis } \\
5 \text { migrations } \\
2 \text { incidents of cholestasis secondary } \\
\text { to FCSEMS placement }\end{array}$ & $\begin{array}{l}\text { Placement of FCSEMSs in patients with refractory benign } \\
\text { pancreatic ductal strictures may be feasible and relatively } \\
\text { safe }\end{array}$ \\
\hline Moon et al. [14] & 32 & $100 \%$ & None & $\begin{array}{l}\text { Effective in resolving pancreatic-duct strictures in chronic } \\
\text { pancreatitis }\end{array}$ \\
\hline $\begin{array}{l}\text { Giacino et al. } \\
{[15]}\end{array}$ & 10 & $90 \%$ & $\mathrm{~N}=2$ cholestasis & $\begin{array}{l}\text { The diameter of the narrowest MPD stricture had increased } \\
\text { significantly from } 3.5 \mathrm{~mm} \text { to } 5.8 \mathrm{~mm} \text { and pain relief was } \\
\text { achieved in nine patients }\end{array}$ \\
\hline Sharaiha et al. & 33 & $96 \%$ & $\begin{array}{l}\mathrm{N}=2 \text { abdominal pain } \\
\mathrm{N}=2 \text { cholestasis }\end{array}$ & Significant improvement in pain and stricture diameter \\
\hline
\end{tabular}

\section{Procedure-related AEs}

Immediate AEs included severe abdominal pain in two patients after stent placement requiring immediate stent removal (both with $10-\mathrm{mm}$ stents), as well as overnight admission for pain control in two patients. Four other patients developed pain that responded to short-interval pain management. Two patients developed elevation in their liver function tests, one of whom required biliary stent placement. No other short-term AEs (<30 days) occurred. There were no cases of pancreatitis, or pancreatic infection. There were no late AEs. One patient had stent impaction at time of removal but the stent was successfully removed endoscopically. There was no stent migration.

\section{Discussion}

$\mathrm{CP}$ is a disease of unrelenting pain flares, exocrine insufficiency, and diabetes. While the exocrine and endocrine pancreatic functions can be replaced, until recently there have been few effective interventions for chronic pain and the treatment emphasis has been and still is on chronic pain management.

Endoscopic therapy in CP is centered on draining the MPD and PD stone fragmentation to provide pancreatic duct decompression. Historically, pancreatic duct strictures have been managed with placement of either single or multiple plastic stents [8, 9]. Recurrent symptomatic strictures limit effectiveness of this approach. FCSEMS were originally developed for palliation of malignant biliary strictures, and subsequently used in benign biliary strictures $[10,11]$. Few studies evaluated use of FCSEMS in symptomatic benign PD strictures [9, 12 -14]. The most recent study included 10 patients with painful chronic pancreatitis and refractory dominant pancreatic duct strictures treated with FCSEMS. They were followed for a relatively long period of 19.8 months. A collaborative international study found use of metal stents feasible, safe, and potentially effec- tive [15]. Our study technical success rate was similarly high when compared to the other four previous studies evaluating FCSEMS in PD strictures ( $\triangleright$ Table 2 ) [12-15]. Prior studies have described acute pancreatitis as a complicating factor in endoscopic management of PD strictures with rates as high as $28 \%$. It was thought to happen because of PD dilation with a stent retriever or a balloon dilator [13]. We report a $0 \%$ incidence of acute pancreatitis post-procedure in this study, highlighting the need to offer appropriate pancreatic duct decompression. Severe abdominal pain occurred in two patients, necessitating stent removal. Those two patients had $10-\mathrm{mm}$ stents as opposed to the $8-\mathrm{mm}$ stents that were placed in other patients. The remainder of the patients who had pain were managed with intravenous narcotics in-house during the first week post-placement. Biliary cholestasis occurred in only $6.1 \%$ of our patients, compared to $15 \%$ and $20 \%$ reported in other studies $[13,15]$. This was treated with biliary stent placement. Our observed post-procedure pain scores were significantly improved after FCSEMS placement, similar to other studies [12$14]$. In this study, the recurrence rate for treated strictures was $0 \%$ after a median follow-up of 8 months (range $5-14$ ), which is significantly better than PD stricture recurrence rates in prior studies that vary from $9 \%$ to $60 \%$ in the same follow-up period $[9,12-14]$. This may be explained by the covering design of the SEMS and the absence of fins, known to be associated with midterm or long-term recurrence [12]. Measurements of PD after FCSEMS placement showed statistically significant improvement inf strictures and a reduction in diameter of the dilated upstream ducts in line with previously reported data [12-15]. Basis for improvement in pain with FCSEMS is unclear and may be related to overall improved pancreatic duct drainage or better stretching of fibrostenotic strictures themselves that cause nerve entrapment and chronic pain.

Limitations of this study include its small size and retrospective nature, which did not allow for collection of all variables. While randomized control trials present a better way to evalu- 
ate a device's effectiveness against the standard of care, in cases of rare events or rare interventions, a multicenter randomized control trial may not be a feasible option. The multicenter nature of this study meant that we could not systematically ensure uniform patient management, which might have been a source of potential bias. We have a remarkably low pancreatitis rate; however, we recognize that this may have been underreported given that we only counted those who were admitted to the hospital. Importantly, while the stricture recurrence rate was $0 \%$, we did note that some patients remained stent-dependent and two patients even required surgery. Our $93 \%$ clinical success rate only shows initial improvement; $36 \%$ of patients still needed interval ERCPs. These patients may be truly refractory to endoscopic therapy. We think that the poor response to pain is due to the fact that the PD strictures in some patients were distal to the metal stent and could not be treated. While VAS is an accepted way to measure pain response, novel research indicates that the Pancreatitis Quality of Life Instrument, a novel validated tool for evaluation of quality of life in chronic pancreatitis, may be useful in future research studies [16].

This is the largest case series of patient with refractory PD strictures and chronic pancreatitis treated with FCSEMS. Although the study concerns a subclass of patients who fail conventional plastic stenting, the long-term follow-up provided is its strength. In addition, it confirms that wide-diameter FCSEMS ( 8 or $10 \mathrm{~mm}$ ) may be difficult to tolerate in $20 \%$ of patients and aggressive management of pain post-deployment may be required.

\section{Conclusion}

In summary, our data confirm previous studies showing that FCSEMS placement for refractory MPD stricture is safe and effective in the majority of patients. Smaller-diameter $(6 \mathrm{~mm})$ FCSEMS are expected to be further investigated through prospective trials before widespread adoption of this type of intervention. In our future prospective studies in chronic pancreatitis, we will be sure to use this tool for a complete assessment of the patients' quality of life, pain, and emotional well-being.

\section{Competing interests}

Drs. Sharaiha, Buscaglia, DiMaio, Siddiqui, and Kahaleh are consultants for Boston Scientific in the area of stent devices. Dr. Buscaglia has received consulting fees from Abbvie (Creon).
References

[1] Majumder S, Chari ST. Chronic pancreatitis. Lancet 2016; 387: 1957 1966

[2] Yadav D, Timmons L, Benson JT et al. Incidence, prevalence, and survival of chronic pancreatitis: a population-based study. Am J Gastroenterol 2011; 106: 2192-2199

[3] Dimagno M], DiMagno EP. Chronic pancreatitis. Curr Opin Gastroenterol 2006: 487-497

[4] van Geenen EJM, Smits MM, Schreuder TCM et al. Smoking is related to pancreatic fibrosis in humans. Am J Gastroenterol 2011; 106: 1161 - 1166 ; quiz 1167

[5] Sakorafas GH, Farnell MB, Nagorney DM et al. Surgical management of chronic pancreatitis at the Mayo Clinic. Surg Clin North Am 2001; 81: $457-465$

[6] Cahen DL, Gouma DJ, Nio Y et al. Endoscopic versus surgical drainage of the pancreatic duct in chronic pancreatitis. N Engl J Med 2007; 356: $676-684$

[7] Sakorafas GH, Tsiotou AG, Peros G. Mechanisms and natural history of pain in chronic pancreatitis: a surgical perspective. J Clin Gastroenterol 2007; 41: 689-699

[8] Gupta R, Reddy DN. Stent selection for both biliary and pancreatic strictures caused by chronic pancreatitis: Multiple plastic stents or metallic stents? J Hepatobiliary Pancreat Sci 2011; 18: 636-639

[9] Shen Y, Liu M, Chen M et al. Covered metal stent or multiple plastic stents for refractory pancreatic ductal strictures in chronic pancreatitis: A systematic review. Pancreatology 2014; 14: 87-90

[10] Kahaleh M, Brijbassie A, Sethi A et al. Multicenter trial evaluating the use of covered self-expanding metal stents in benign biliary strictures: time to revisit our therapeutic options? J Clin Gastroenterol 2013; 47: 695-699

[11] Devière J, Reddy DN, Püspök A et al. Successful management of benign biliary strictures with fully covered self-expanding metal stents. Gastroenterology 2014; 147: 385-395

[12] Sauer B, Talreja J, Ellen K et al. Temporary placement of a fully covered self-expandable metal stent in the pancreatic duct for management of symptomatic refractory chronic pancreatitis: preliminary data (with videos). Gastrointest Endosc 2008; 68: 1173-1178

[13] Park DH, Kim MH, Moon SH et al. Feasibility and safety of placement of a newly designed, fully covered self-expandable metal stent for refractory benign pancreatic ductal strictures: a pilot study (with video). Gastrointest Endosc 2008; 68: 1182-1189

[14] Moon SH, Kim MH, Park DH et al. Modified fully covered self-expandable metal stents with antimigration features for benign pancreaticduct strictures in advanced chronic pancreatitis, with a focus on the safety profile and reducing migration. Gastrointest Endosc 2010; 72: $86-91$

[15] Giacino C, Grandval P, Laugier R. Fully covered self-expanding metal stents for refractory pancreatic duct strictures in chronic pancreatitis. Endoscopy 2012; 44: 874-877

[16] Wassef W, John D, Kathleen M et al. Pancreatitis Quality of Life Instrument: A Psychometric Evaluation. Am J Gastroenterol 2016; 111: $1177-1186$ 